\title{
Fuerza de adhesión en dentina intracameral tratada con diferentes irrigantes endodónticos.
}

\section{Joaquín Vega Montoya ${ }^{1^{*}}$}

1. Departamento Odontología Restaurativa, Facultad de Odontología, Universidad Nacional Autónoma de Nicaragua, León (UNAN-LEÓN).

RESUMEN

El objetivo de esta investigación fue, evaluar la fuerza de adhesión de tres sistemas adhesivos: Single Bond 2, One Step plus y PQ1, en dentina intracameral previamente irrigada con $\mathrm{NaOCl} 3 \%$ y NaOCl 3\% + EDTA 17\% combinados; por medio de la prueba microtensil. Se seleccionaron 90 terceros molares humanos libres de caries, los cuales se dividieron en tres grupos ( $\mathrm{N}=30)$ dependiendo del irrigantes endodóntico; GI: Agua destilada (control), GII: NaOCl 3\% y GIII: NaOCl 3\% + EDTA 17\%. Cada grupo se subdividió en tres subgrupos dependiendo del sistema adhesivo a utilizar. A los dientes se les colocó resina compuesta Z100 e inmediatamente después se seccionaron en sentido trasversal hasta obtener un espécimen por diente de la interface adhesiva, con un área aproximada de $1 \mathrm{~mm}$ * $1 \mathrm{~mm}$. Cada espécimen fue sometido a la prueba adhesiva microtensil, en una máquina de ensayos universal (Tinius Olsen H10K-S). Los valores de la fuerza adhesiva fueron expresados en MPa y analizados con un análisis de varianza (ANOVA) de dos vías, a través de la prueba estadística Scheffé utilizando el programa estadístico KaleidaGraph 4.0. a 0.05 grados de significancia. Dentro de las condiciones en las que se realizó el presente estudio se pudo concluir que la fuerza de adhesión microtensil de los adhesivos Single Bond 2, One Step Plus y PQ1 fueron estadísticamente iguales entre sí para el mismo irrigante y cuando se trata la dentina intracameral con irrigantes endodónticos como el $\mathrm{NaOCl} 3 \%$ o NaOCl 3\% + EDTA 17\% en combinación; la fuerza de adhesión microtensil de los adhesivos Single Bond 2, One Step Plus y $\mathrm{PQ1}$ disminuye.

Palabras claves: Adhesión, dentina intracameral, irrigantes endodónticos.

\section{INTRODUCCIÓN}

Los dientes que han sido tratados endodónticamente pierden generalmente estructura debido a trauma, caries y a los procedimientos endodónticos en si ${ }^{[1]}$.

La restauración de estos dientes está sujeta a mucha controversia, especialmente en lo que respecta a la resistencia a la fractura. Antiguamente, se usaba amalgama como restauración definitiva directa. Actualmente los conceptos restaurativos han cambiado, utilizando tecnología adhesiva como mejor opción ${ }^{[2]}$.

Cuando se utiliza resina compuesta como material restaurador, los dientes deben tener suficiente estructura; a tal punto, que algunos sólo necesitan el sellado del esmalte y dentina que fue removida por el procedimiento endodóntico ${ }^{[1]}$.

Las ventajas de la restauraciones con resinas compuestas son: conservación del tejido dentario, capacidad de adhesión a dentina y esmalte, aumento de la resistencia a la fractura de las estructuras remanentes, aumento de la retención de la restauración y reducción de la microfiltración; todas estas ventajas se han llevado a cabo por el uso de sistemas adhesivos que se unen micromecánicamente con la dentina previamente acondicionada con grabado ácido total ${ }^{[1,3,4]}$.

\footnotetext{
*Autor para correspondencia: vegamontoya@hotmail.com
}

El éxito longitudinal de las restauraciones en los dientes tratados endodónticamente depende de la calidad estructural, estética de la restauración, de su adaptación clínica y de la salud de los tejidos de soporte, pero también del pronóstico de la reconstrucción ${ }^{[5]}$.

Aunque, se ha avanzado enormemente en la restauraciones de estos dientes la carencia de sellado y adhesión entre la restauración final y la estructura dental es un efecto adverso del tratamiento endodóntico. Esta carencia de sellado puede afectarse por muchos factores, dentro de ellos se encuentran los irrigantes químicos usados durante la limpieza y conformación de los conductos radiculares que puede alterar la composición superficial de la dentina intracameral y radicular afectando la interacción con el material utilizado para el selle coronal[6].

El sellado coronal posterior al tratamiento de conductos es de vital importancia para prevenir la temprana filtración marginal, contribuyendo positivamente en el pronóstico de los tratamientos endodónticos ${ }^{[5,7]}$.

Con las limitaciones del presente estudio se tuvo la finalidad de comparar la fuerza de adhesión entre tres sistemas adhesivos con las paredes de dentina intracameral irrigada previamente con diferentes soluciones endodónticas. 


\section{MATERIALES Y MÉTODOS.}

\section{Muestra.}

Para el presente estudio experimental (in vitro) se utilizaron 90 terceros molares inferiores, libres de caries, almacenados en agua destilada y extraídos en un plazo no mayor de 6 meses ${ }^{[8,9]}$.

Los dientes se dividieron en 3 grupos $(n=30)$, según los irrigantes empleados (un control y dos irrigantes endodónticos); cada grupo se subdividió en tres subgrupos $(n=10)$ dependiendo del sistema adhesivo a usar.

\section{Técnica de corte dentario.}

Cada tercer molar se cortó a la mitad en sentido mesiodistal y corono apical (verticalmente); tomando como referencia el tercio medio de cada pieza, para exponer los cuernos pulpares, el techo y piso de la cámara pulpar. Se realizaron dos cortes adicionales; el primero, $0.5 \mathrm{~mm}$ apical al techo de la cámara pulpar en sentido mesio distal y horizontalmente; el segundo, $0.5 \mathrm{~mm}$ coronal al piso pulpar. El corte se realizó con una sierra de disco de diamante de baja velocidad (isomet slow speed saw, BUEHLER) para dejar expuesta las paredes de la cámara pulpar ${ }^{[8,9]}$. (Figura 1 y 2).

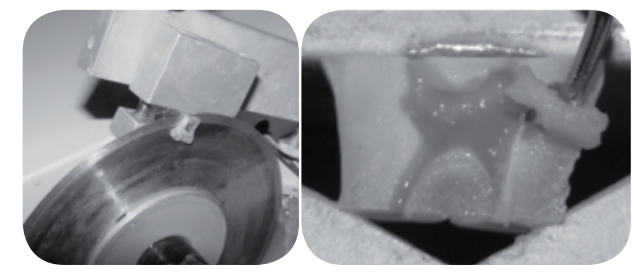

Figura 1 y 2. Realización del corte dentario hasta exponer la pulpa cameral.

Con la cavidad pulpar expuesta, se eliminó cuidadosamente los restos de pulpa dental utilizando un instrumento manual de extremo cortante, tratando de no dañar las paredes de la dentina (Figura 3 y 4). Luego se colocaron los cortes dentarios en un aparato ultrasónico (Vitasonic II) de limpieza por diez minutos.

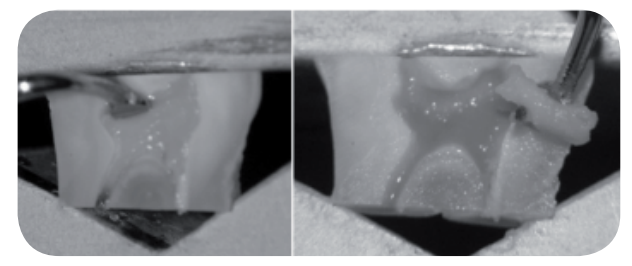

Figura 3 y 4. Eliminación de la pulpa cameral.

\section{Técnica de irrigación.}

Luego de limpiar la cámara pulpar de cada diente, se sumergió en $10 \mathrm{ml}$ agua destilada para el grupo control y $10 \mathrm{ml}$ de solución endodóntica; de acuerdo al grupo al que pertenecían, como se detalla a continuación:

- Grupo No 1 ( $n=30)$; agua destilada (control) por 15 minutos.

-Grupo No 2 ( $\mathrm{n}=30)$; hipoclorito de sodio $(\mathrm{NaOCl}$, Macrodin ${ }^{\circledR}$ cloro concentrado al 3\%) por 15 minutos.

- Grupo No 3 ( $n=30)$; Hipoclorito de sodio $(\mathrm{NaOCl}$, Macrodin ${ }^{\circledR}$ Cloro concentrado al 3\%) por 15 min.+ EDTA al $17 \%$ por 5 minutos.

Después del procedimiento anteriormente detallado, las paredes de la cámara pulpar se lavaron con 10 $\mathrm{ml}$ de agua destilada a temperatura ambiente y se secaron con aire por 5 segundos. El lavado servía para remover restos de la sustancia utilizada o predentina desprendida por efecto del irrigante $\mathrm{e}^{[2,6]}$.

\section{Aplicación del sistema adhesivo.}

Una vez realizada la irrigación, se procedió a dividir cada grupo en tres subgrupos $(n=10)$. A cada subgrupo se le asignó un adhesivo diferente.

1-Adper ${ }^{\mathrm{TM}}$ Single Bond 2 (SB2). 3M ESPE.

2-One-Step ${ }^{\circledR}$ Plus (OSP). Bisco, Inc.

3-PQ1 ${ }^{\circledR}$ (PQ1). Ultradent Product, Inc.

\section{Adhesivo Adper ${ }^{\mathrm{TM}}$ Single Bond 2 (SB2). 3M ESPE.}

Se realizó el acondicionamiento ácido de las paredes de la cámara pulpar con ácido fosfórico al 35\% (Singlebond $^{\mathrm{TM}}$ Etchant Phosphoric Acid, 3M ESPE) por 15 segundos (Figura 5), se irrigó con agua por 30 segundos y secó pasando gasas estériles sobre la superficie (Figura 6), el Adper ${ }^{\mathrm{TM}}$ Single Bond 2 (SB2) 3M ESPE (Figura 7), es un sistema adhesivo con solvente a base de etanol y agua, se aplicó enérgicamente dos capas con un Micro Brush y adelgazó cada capa con un chorro de aire (Figura 8), luego se fotopolimerizó por 20 segundos cada capa con una lámpara Hilux Optimax (Curing Light Unit) calibrada entre 450-500 nm

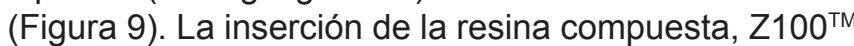
Restaurative (3M ESPE); se realizó con un instrumento manual (Hu-Friedy) para resina (Figura 10), colocando capas de $2 \mathrm{~mm}$ de grosor para sellar la cámara pulpar; cada capa se fotopolimerizó por 40 segundos con Hilux Optimax (Curing Light Unit) calibrada entre 450-500 $\mathrm{nm}$, para asegurar el completo curado del composite. Se utilizó la cantidad de incrementos necesarios hasta obtener el volumen adecuado de resina (Figura 11). 


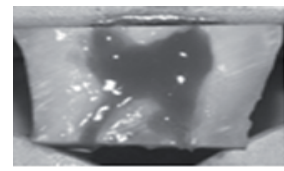

Figura 5. Acondicionamiento ácido por 15 segundos.

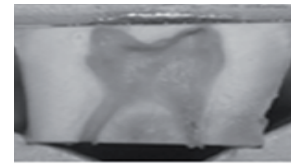

Figura 6. Secado de la superficie.

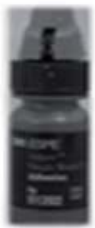

Figura 7. Adper ${ }^{T M}$ Single Bond 2 (SB2) 3M ESPE

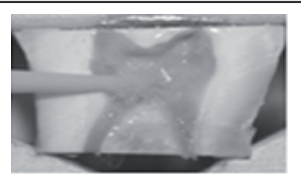

Figura 8. Aplicación del sistema adhesivo

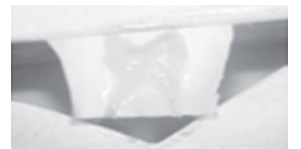

Figura 9. Fotopolimerización por 20 segundos.

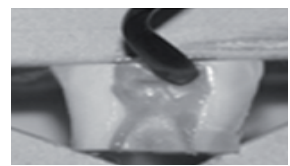

Figura 10. Inserción de resina compuesta

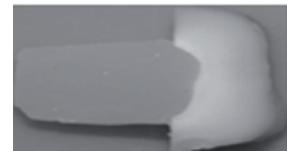

Figura 11. Listo para la fabricación de los especímenes.

Adhesivo One-Step Plus (OSP) Bisco, inc.

Se realizó el acondicionamiento ácido de las paredes de la cámara pulpar con ácido fosfórico al 35\% (Singlebond $^{\mathrm{TM}}$ Etchant Phosphoric Acid, 3M ESPE) por 15 segundos (Figura 12), se irrigó con agua por 30 segundos y secó con gasas estériles sobre la superficie (Figura 13). El sistema adhesivo One-Step Plus ${ }^{\circledR}$ (Bisco) con solvente de acetona (Figura 14), se agitó previamente y se aplicó en dos capas con un aplicador (Micro Brush), frotando enérgicamente (Figura 15), seguido de un chorro de aire para adelgazar cada capa, luego se fotopolimerizó por 20 segundos con una lámpara Hilux Optimax (Curing Light Unit) calibrada entre 450-500 nm (Figura 16). La inserción de la resina compuesta, $\mathrm{Z}^{100^{\mathrm{TM}}}$ Restaurative (3M ESPE); se realizó con un instrumento manual (Hu-Friedy) para resina (Figura 17), colocando capas de $2 \mathrm{~mm}$ de grosor para sellar la cámara pulpar; cada capa fue fotopolimerizada por 40 segundos con Hilux Optimax (Curing Light Unit) calibrada entre $450-500 \mathrm{~nm}$, para asegurar el completo curado del composite. Se utilizó la cantidad de incrementos necesarios hasta obtener el volumen adecuado de resina (Figura 18).

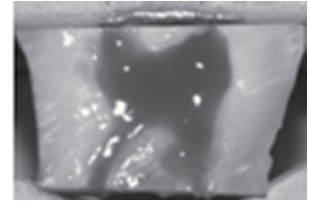

Figura 12. Acondicionamiento ácido por 15 segundos.

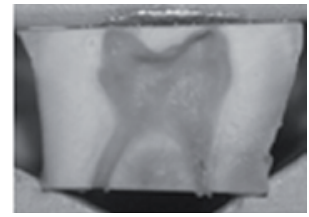

Figura 13. Secado de la superficie.

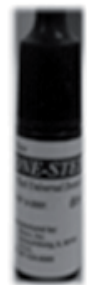

Figura 14. One-Step ${ }^{\circledR}$ Plus (OSP). Bisco, Inc.

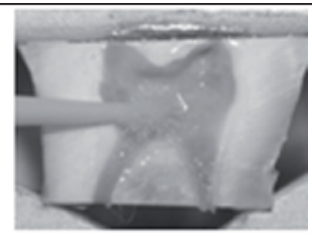

Figura 15. Aplicación del sistema adhesivo

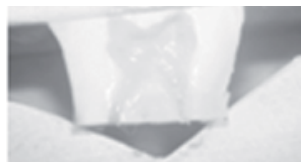

Figura 16. Fotopolimerización por 20 segundos.

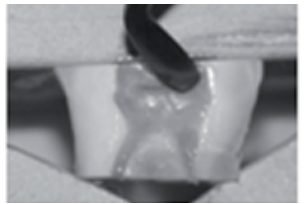

Figura 17. Inserción de resina compuesta

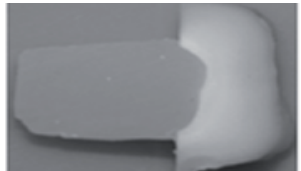

Figura 18. Listo para la fabricación de los especímenes. 


\section{Adhesivo PQ1 ${ }^{\circledR}$ (PQ1), Ultradent Product, Inc.}

Se realizó el acondicionamiento ácido de las paredes de la cámara pulpar con ácido fosfórico al 35\% (Singlebond ${ }^{\mathrm{TM}}$ Etchant Phosphoric Acid, 3M ESPE) por 15 segundos (Fig. 19), se irrigó con agua por 30 segundos y secó pasando gasas estériles sobre la superficie (Figura 20). EI PQ1 ${ }^{\circledR}(P Q 1)$ Ultradent Product, Inc. (Figura 21), es un sistema adhesivo con solvente de alcohol; de jeringa única, el que se aplicó enérgicamente dos capas con la jeringa dentoinfusora y adelgazando cada capa con un chorro de aire, se fotopolimerizó por 20 segundos cada capa con una lámpara Hilux Optimax (Curing Light Unit) calibrada entre 450-500 nm (Figura 22). La inserción de la resina compuesta, Z100 ${ }^{\mathrm{TM}}$ Restaurative (3M ESPE); se realizó con un instrumento manual (HuFriedy) para resina (Figura 23), colocando capas de $2 \mathrm{~mm}$ de grosor para sellar la cámara pulpar; cada capa se fotopolimerizó por 40 segundos con Hilux Optimax (Curing Light Unit) calibrada entre $450-500 \mathrm{~nm}$, para asegurar el completo curado del composite. Se utilizó la cantidad de incrementos necesarios hasta obtener el volumen adecuado de resina (Figura 24).

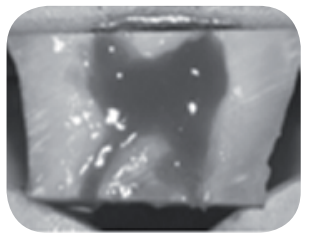

Figura 19. Acondicionamiento ácido por 15 segundos.

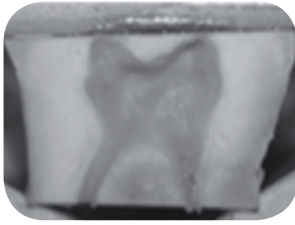

Figura 20. Secado de la superficie.

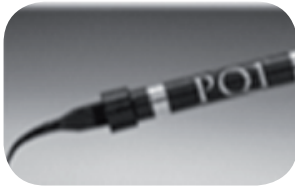

Figura 21. $P Q 1^{\circledR}(P Q 1)$. Ultradent Product, Inc

\section{Fabricación de la Muestra.}

Después de agregar resina compuesta sobre la cámara pulpar, se procedió a cortar con una sierra diamantada de baja velocidad (isomet slow speed saw, BUEHLER); con abundante irrigación, trozos de $1 \mathrm{~mm}$ por $1 \mathrm{~mm}$ de área a nivel de las paredes de la cámara pulpar. El corte se realizó en sentido vestíbulo lingual (horizontal), abarcando dentina y resina compuesta (Figura 25).

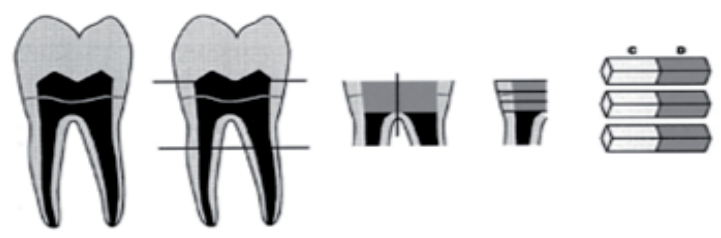

Figura 25. Representación esquemática para la obtención de especímenes.

A. Corte sentido mesio-distal de los dientes B. Corte de cámara pulpar

C.Colocación de resina intracameral

D. Corte de los especímenes

E.Especímenes terminados $1 \mathrm{~mm}$ * $1 \mathrm{~mm}$ de área (c:composite, $d$ : dentina)
Las dimensiones exactas fueron calibradas cuidadosamente usando un micrómetro digital (Digimatic Solar, Mitutoyo Corp., Tokyo, Japan) con una precisión de $0.01 \mathrm{~mm}$.

Cada espécimen fue rotulado según los tipos de irrigantes endodónticos y el sistema adhesivo usado; posteriormente, se realizó la prueba adhesiva (microtensil) con la máquina de pruebas universal Tinius Olsen H1OK-S con aditamentos especiales (Fig. 26). La obtención de las muestras se realizó en un ambiente con temperatura y humedad controladas. La temperatura se mantuvo a $23 \pm 2^{\circ} \mathrm{C}$ con humedad relativa de $50 \pm 5 \%$ según lo indica la ISO/TS No. 11405 (2003), esta fue controlada por medio de una unidad central de temperatura digitalizada. Los especímenes fueron obtenidos entre 2 p.m. y 4 p.m. para asegurar las condiciones constantes del ambiente de trabajo. 


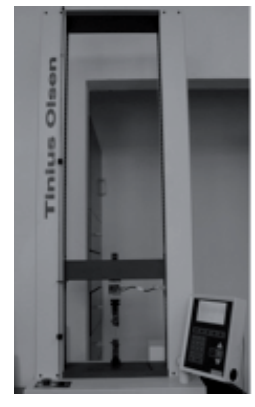

Figura 26. Tinius Olsen H1OK-S.

\section{Prueba adhesiva}

Los especímenes fueron fijados cuidadosamente en sus extremos a un dispositivo de prueba, utilizando para esto pegamento cianoacrilato (Loctite Super Bonder®, Henkel. Brazil). Luego de fijar los especímenes al dispositivo de prueba, cada uno estos se colocó verticalmente y se fijó en los aditamentos de la máquina de prueba universal Tinius Olsen H10K-S (Figura 27); se realizó la tensión y se calculó la falla adhesiva hasta que el espécimen se rompió (Figura 28). Cada falla fue expresada en megapascales (MPa).

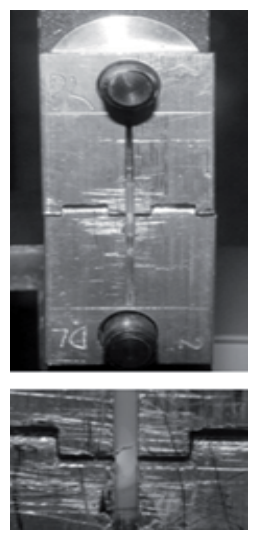

Figura 27. Fijación y colocación de los especímenes en la máquina Tinius Olsen H1OK-S.

Figura 28. Espécimen fallado.

\section{Análisis estadístico}

Se calcularon los promedios y las desviaciones estándar de la prueba micro-tensil de adhesión, se hizo el análisis de varianza (ANOVA) de dos vías (material adhesivo, irrigantes endodónticos), calculado a un nivel de significancia de 0.05 .

Se compararon los promedios con la prueba estadística Scheffé utilizando el programa estadístico KaleidaGraph 4.0. a 0.05 grados de significancia.

\section{RESULTADOS Y DISCUSIÓN.}

Para el estudio se prepararon 90 modelos de prueba, de los cuales 14 se fracturaron antes de la realización de la prueba microtensil, por lo tanto los resultados se obtuvieron de un total de 76 especímenes.

Los resultados promedios obtenidos de la prueba microtensil donde se utilizaron tres sistemas adhesivos (SB2, OSP y PQ1) en dentina intracameral tratada con diferentes irrigantes endodónticos (agua destilada, hipoclorito de sodio 3\% e Hipoclorito de sodio 3\% y EDTA $17 \%$ en combinación) se detallan en la tabla 1 y se grafican separadamente a continuación.

Tabla 1. Resultados de la prueba microtensil (MPa) de los diferentes sistemas adhesivos sobre dentina intracameral tratada con diferentes irrigantes.

\begin{tabular}{l|ccc}
\hline Adhesivo & Agua destilada & Hipoclorito 3\% & Hipoclorito3\% +EDTA17\% \\
SB2 & $20,4(7,1)$ & $14,9(4,7)$ & $18,8(2,7)$ \\
OSP & $28,7(9,6)$ & $17,4(3,7)$ & $14(5,6)$ \\
PQ1 & $23,3(7,6)$ & $14,7(5,9)$ & $16,4(5,8)$ \\
\hline
\end{tabular}

SB2, Single Bond 2; OSP, One Step Plus; PQ1.

* Desviación Estandar entre paréntesis

En la tabla 1 se puede observar de manera general que los mayores niveles de adhesión se encuentran en los tres grupos de adhesivos que interactuaron con el irrigante control (agua destilada), siendo el mayor 28,7 MPa del sistema adhesivo One Step Plus (OSP); teniendo una desviación estándar de 9,6. Aunque el resultado fue mayor al obtenido por el adhesivo SB2 (20,4 MPa) y el PQ1 (23,3 MPa), esta diferencia no fue estadísticamente significativa cuando se analizan con el mismo irrigante (tabla 1).

Si se analiza desde el punto de vista individual los adhesivos tuvieron promedios globales de adhesión de $18 \mathrm{MPa}$ para los adhesivos SB2 y PQ1 y $20 \mathrm{MPa}$ para el OSP. Si comparamos los resultados de adhesión según los irrigantes utilizados se puede ver que los grupos del hipoclorito al 3\%, hipoclorito al 3\% y EDTA $17 \%$ combinado; reportan los niveles de adhesión más bajos siendo el menor de $14 \mathrm{MPa}$ para el adhesivo OSP, aun así estos datos no muestran diferencias estadísticas entre los adhesivos del mismo grupo según irrigante.

Los promedios globales de adhesión microtensil según los irrigantes fueron: $24 \mathrm{MPa}$ para los adhesivos cuando la dentina fue irrigada con agua destilada y 16 $\mathrm{MPa}$ cuando fue irrigada con $\mathrm{NaOCl} 3 \%$ y se repite el valor de $16 \mathrm{MPa}$ cuando se irrigó con $\mathrm{NaOCI} 3 \%+$ EDTA $17 \%$ combinado. Esta diferencia fue estadísticamente significativa según como se detalla a continuación. 
En la tabla 2 donde se realiza el análisis de varianza de dos vías para los adhesivos e irrigantes endodónticos, no se encontraron diferencias estadísticas significativas en el promedio de la fuerza microtensil entre los adhesivos $(p=0.705)$, pero si se encontró diferencias entre los irrigantes $(p<0.0001)$. Por lo tanto, existe suficiente evidencia para no rechazar la hipótesis nula; por lo tanto el promedio de la fuerza de adhesión es igual estadísticamente entre los adhesivos cuando la dentina de la cámara pulpar es tratada previamente con el mismo irrigante endodóntico.

Tabla 2. Resultados del análisis de varianza (ANOVA) de dos vías, según material adhesivo e irrigantes endodónticos

\begin{tabular}{|l|c|c|c|c|c|}
\hline \multicolumn{1}{|c|}{ Source } & DF & SS & MS & F & P \\
\hline Total & 53 & $1.585,3333$ & 29,91195 & & \\
Adhesivo & 2 & 13 & 6,5 & 0,3518443 & 0,70531 \\
Irrigante & 2 & 654,33333 & 327,16667 & 17,709503 & $<0,0001$ \\
Interacción & 4 & 86,666667 & 21,666667 & 1,1728148 & 0,33552 \\
Error & 45 & 831,33333 & 18,474074 & & \\
\hline
\end{tabular}

Fuente: Base de datos KaleidaGraph 4.0.

Debido a la diferencia encontrada en la variable "irrigante" se realizó el test de Scheffé (tabla 3) para saber en qué grupo existía verdadera diferencia estadística. Se encontró diferencia estadísticamente significativa entre agua destilada e hipoclorito 3\% +EDTA 17\% ( $p<0,0001)$ y entre agua destilada e hipoclorito $3 \% \quad(p<0,0001)$, pero no entre $\mathrm{NaOCl}$ y EDTA.

Tabla 3. Test Scheffé según los irrigantes endodónticos del estudio.

\begin{tabular}{|l|c|c|c|c|c|}
\hline \multicolumn{1}{|c|}{ Source } & DF & SS & MS & F & P \\
\hline Total & 53 & $1.585,3333$ & 29,91195 & & \\
Adhesivo & 2 & 13 & 6,5 & 0,3518443 & 0,70531 \\
Irrigante & 2 & 654,33333 & 327,16667 & 17,709503 & $<0,0001$ \\
Interacción & 4 & 86,666667 & 21,666667 & 1,1728148 & 0,33552 \\
Error & 45 & 831,33333 & 18,474074 & & \\
\hline
\end{tabular}

Fuente: Base de datos KaleidaGraph 4.0.

En la figura 29 se observa que el promedio de la fuerza de adhesión microtensil del Adper Single Bond 2 decrece según el irrigante utilizado para tratar la dentina.

El valor más elevado se logró con el agua destilada (control) obteniendo un nivel de adhesión de 20,4 MPa; en comparación con el hipoclorito $3 \%+E D T A 17 \%$ con un valor intermedio de 18,8 MPa de adhesión y 14,9 MPa con el hipoclorito $3 \%$ siendo éste el menor. Se tiene en cuenta que esta comparación es estadísticamente significativa.

En la figura 30 se observa que la fuerza de adhesión microtensil del sistema adhesivo One Step Plus decrece, según el irrigante utilizado para tratar la dentina.

El mejor resultado se logró con el agua destilada (control) obteniendo un nivel de adhesión de 28,7 MPa; en comparación con el hipoclorito $3 \%$ e hipoclorito $3 \%+E D T A 17 \%$ con valores de $17,4 \mathrm{MPa}$ y $14 \mathrm{MPa}$ respectivamente. Esta comparación al igual que el anterior es estadísticamente significativa.

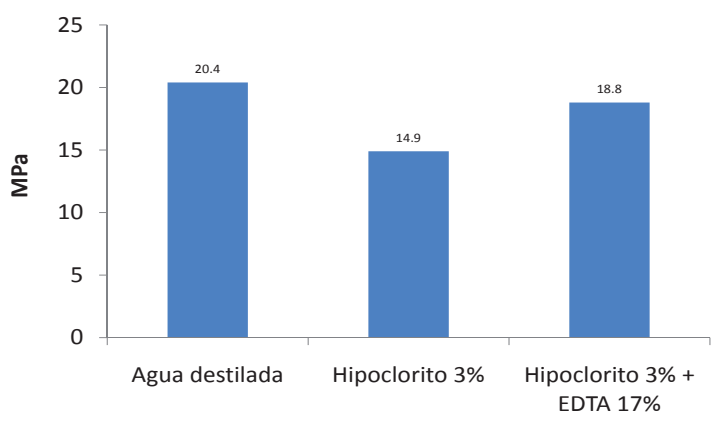

Figura 29. Fuerza de adhesión microtensil del adhesivo Adper Single Bond 2, según irrigante (MPa).

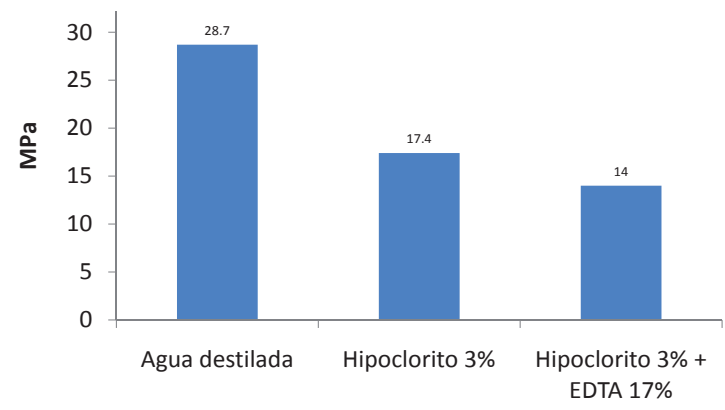

Figura 30. Fuerza de adhesión microtensil del adhesivo One Step Plus, según irrigante (MPa). 


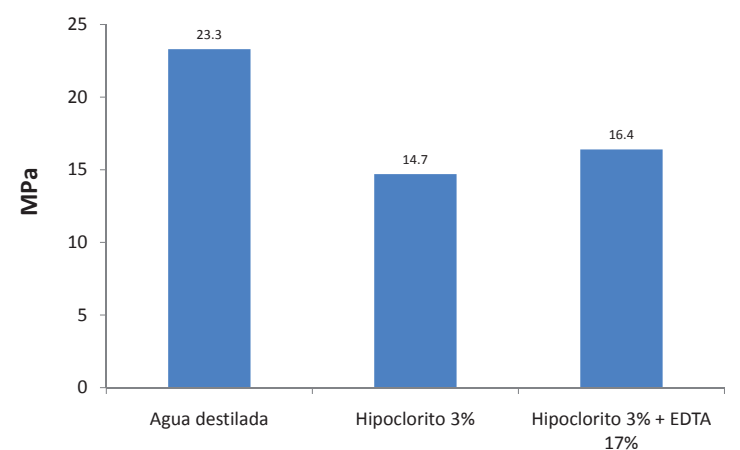

Figura 31. Fuerza de adhesión microtensil del adhesivo PQ1, según irrigante (MPa).

En la figura 31 se puede observar que la fuerza de adhesión microtensil del sistema adhesivo PQ1 decrece, según el irrigante utilizado para tratar la dentina. El nivel más elevado de adhesión resultó con agua destilada (control) obteniendo un resultado de 23,3 MPa, en comparación con el hipoclorito $3 \%$ e hipoclorito $3 \%+E D T A 17 \%$ con valores de $14,7 \mathrm{MPa}$ y $16,4 \mathrm{MPa}$ respectivamente, valores que indican una diferencia estadística.

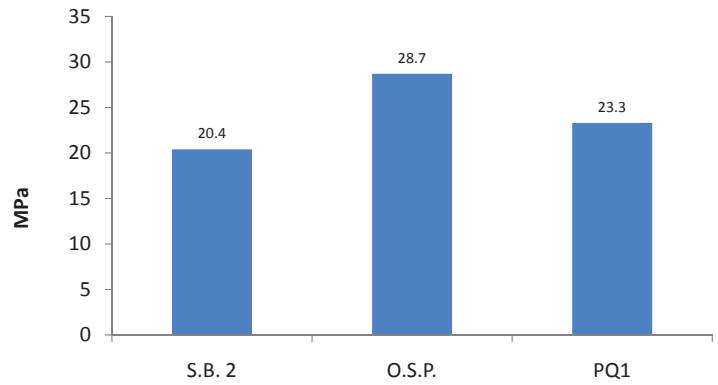

Figura 32. Fuerza de adhesión microtensil de los adhesivos dentinarios, según agua destilada (MPa).

La figura 32 muestra la fuerza de adhesión de los sistemas adhesivos del estudio cuando interactúan con agua destilada (control).El adhesivo con mayor fuerza de adhesión fue el One Step Plus (O.S.P) con 28,7 MPa y en orden decreciente el PQ1 y Single Bond (S.B) con 23,3 y 20,4 MPa. Aunque exista diferencia en MPa estos resultados no son diferentes estadísticamente.

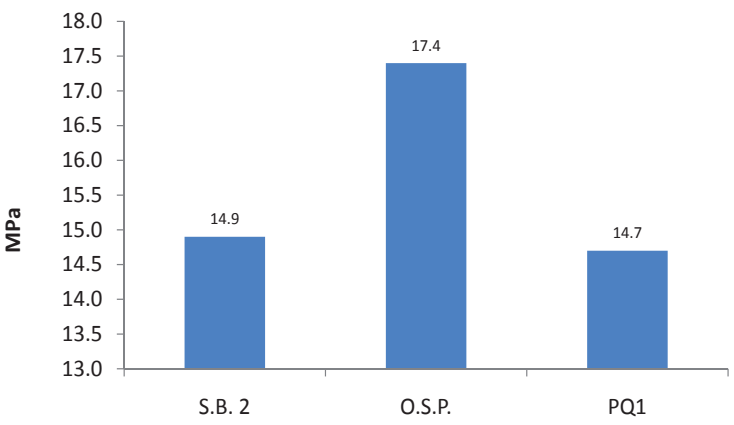

Figura 33. Fuerza de adhesión microtensil de los adhesivos dentinarios, según Hipoclorito 3\% (MPa).
La figura 33 se compara la fuerza de adhesión de los sistemas adhesivos del estudio con el irrigante hipoclorito de sodio al 3\%, en el cual se obtuvo que el One Step Plus mostró los mejores resultados con 17,4 MPa y resultados similares para los adhesivos Single Bond y PQ1 14,9 y 14,7 MPa respectivamente. Al igual que los datos anteriores los resultados no son diferentes estadísticamente.

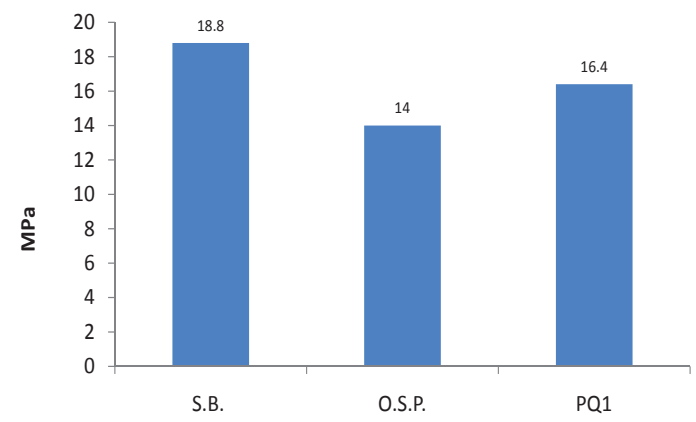

Figura 34. Fuerza de adhesión microtensil de los adhesivos dentinarios, según Hipoclorito 3\% +EDTA17\% (MPa).

La figura 34 se observa claramente los niveles de adhesión de los tres sistemas adhesivos del estudio tratados con hipoclorito y EDTA en conjunto, los cuales mostraron niveles de 18,8 MPa para el Single Bond, $14 \mathrm{MPa}$ para el One Step Plus y 16,4 MPa en PQ1, considerados iguales estadísticamente.

Los resultados del estudio indican que cuando se usan los sistemas adhesivos: Adper Single Bond 2, One Step Plus y PQ1 sobre dentina intracameral, la fuerza de adhesión se ve disminuida por irrigantes endodónticos como el hipoclorito de sodio 3\% y EDTA $17 \%$ solos o en combinación. Por el contrario el agua destilada (control) no afecta la fuerza de adhesión en dentina intracameral con los mismos adhesivos. Esto también fue probado por Belli y col, Inue y col, Ozturk y col. en pruebas similares.

El hipoclorito de sodio y el EDTA son extensamente utilizados para la realización de la limpieza y conformación de los conductos radiculares en la terapia endodóntica ya que proveen desinfección y lubricación de los tejidos [6, 10, 11]. El $\mathrm{NaOCl}$ aunque es útil para eliminar los componentes del tejido pulpar, puede disminuir las propiedades físicas y mecánicas de la dentina. Éste se descompone en cloruro de sodio y en oxígeno, lo que podría proporcionar la oxidación de algunos componentes de la dentina; por consiguiente, la oxidación de la matriz de colágeno de la dentina disminuyendo su módulo de elasticidad y fuerza de la flexión. También podría afectar la penetración de los sistemas adhesivos a la estructura de la dentina y la polimerización de los monómeros en la desmineralizada 
dentina, por lo que podría influir en la calidad de las restauraciones ${ }^{[12,13]}$.

Algunos estudios se han llevado a cabo para evaluar la interacción entre algunos sistemas adhesivos y la dentina de la cámara pulpar previamente sometida a tratamiento con $\mathrm{NaOCl}$. Independiente del sistema adhesivo y la concentración del $\mathrm{NaOCl}$, siempre tiende a disminuir la fuerza de adhesión, reportando una disminución de hasta $23 \%$ de la fuerza de adhesión; se cree que ésta pérdida en dicha fuerza se debe al cambio en la estructura dentinal[3,12].

Ozturk y col., reportaron mejores resultados cuando se utilizaban adhesivos autoacondicionadores que adhesivos de acondicionamiento ácido previo, debido a que estos autoadhesivos poseen en su composición ácidos débiles por lo tanto provocan un cambio menor en la estructura de la paredes de la dentina, que los ácidos fuertes de los adhesivos que utilizan grabado total. Pero la tendencia siempre fue; al igual que este estudio, la disminución de la adhesión ${ }^{[3]}$. En el presente estudio la adhesión tuvo la tendencia a disminuir respecto a los irrigantes ( $\mathrm{NaOCl} 3 \%$ y EDTA $17 \%$ ) aunque no se realizó una comparación entre adhesivos de diferente naturaleza ya que todos los adhesivos que se utilizaron pertenecían a la misma clasificación.

La pérdida de la fuerza de adhesión podría ser recuperada cuando la irrigación con $\mathrm{NaOCl}$ fue seguida por ácido ascórbico al 10\% o ascórbico de sodio al $10 \%$ según reporta Vongphan y col. ${ }^{[1]}$. Sin embargo, una prueba mecánica de adhesión no puede evaluar los cambios causados por $\mathrm{NaOCl}$ en la estructura de la dentina.

El EDTA al $17 \%$ es muy efectivo para eliminar el "barro dentinario" e incrementa el diámetro de los túbulos dentinarios. Al igual que el $\mathrm{NaOCl}$ el EDTA provoca cambios en la estructura de la dentina como en la microdureza, permeabilidad y solubilidad. Estos cambios se realizan en la proporción de contenido mineral superficial (calcio/fosfato) por lo que se puede afectar la habilidad de la dentina para aprovechar los adhesivos $^{[11]}$.

En el presente estudio 30 de los especímenes fueron irrigados con EDTA $17 \%$ por 5 minutos posterior a la irrigación con $\mathrm{NaOCl}$ por 15 minutos para hacer la prueba más cercana a la práctica clínica. Los resultados que se obtuvieron fue una disminución de la fuerza de adhesión de los sistemas adhesivos en comparación con el irrigante control.
Nascimento y col. reportaron niveles bajos de adhesión del sistema Clearfil SE Bond, cuando se utilizó en dentina intracameral tratada previamente con $\mathrm{NaOCl}$ $5.25 \%$ y $\mathrm{NaOCl} 5.25 \%$ +EDTA $17 \%{ }^{[6]}$. Este estudio solamente comparó el mismo adhesivo con diferentes irrigantes endodónticos, pero la tendencia fue la misma reportada en el presente estudio, que los soluciones endodónticas influyen negativamente en la adhesión.

Los adhesivos utilizados en este estudio se clasificaron dentro del grupo que necesitan acondicionamiento ácido previo, con solventes diferentes y con un porcentaje de relleno distinto, siendo el PQ1 el que más relleno contenía. Debido a estas características se pensó que el nivel de adhesión sería similar entre los adhesivos, lo cual se comprobó.

Belli, reportó mejores niveles de adhesión del One Step, que los resultados del One Step Plus en este estudio. La adhesión en el estudio de Belli y col. se realizó en diferentes regiones del la cámara pulpar tratada previamente con $\mathrm{NaOCI} 5 \%$, estos buenos resultados se los atribuye a la cantidad y tamaño del relleno del sistema adhesivo. Pero como se detalló anteriormente una prueba mecánica no puede explicar el efecto del irrigante sobre la dentina ${ }^{[7]}$.

Esta prueba microtensil consiste en evaluar muestras de unión entre resina y dentina en áreas aproximadas a $1 \mathrm{~mm}^{2}$, la dificultad real para la realización de los especímenes es el tamaño; ya que esta área es tan pequeña que pueden ocurrir eventos catastróficos (fracturas prematuras) que no permitan realizar la prueba. Por lo que se deben realizar con sumo cuidado. Sin embargo solo el hecho de la vibración generada por la sierra diamantada cuando se realiza el corte podría provocar estas fallas. Por lo que se debe tener siempre presente que aunque es una excelente prueba adhesiva, tiene sus limitantes y desafíos.

Cuando se realiza un experimento como este se tratan de controlar la mayor cantidad de variables posibles, pero siempre se dejan otras por fuera y puede ser posible que si esas otras variables se estudiaran podrían proporcionar resultados diferentes. Toda prueba de laboratorio tiene como objetivo brindar una información al profesional, en este caso brindar la información sobre la adhesión en dentina intracameral tratada con diferentes irrigantes endodónticos; pero siempre los resultados distan de la realidad clínica aunque pueden servir como un punto de partida.

Estudios in vitro realizados en la UCR (Universidad de Costa Rica), que refieren que los remanentes del eugenol dejados por los cementos selladores 
de endodoncia en al dentina radicular, inhiben la polimerización de los materiales resinosos y por ende disminuye la adhesión. Respecto a lo anterior, Loaiza, afirma que los selladores de los conductos radiculares formulados con eugenol, no influyen estadísticamente en la fuerza de adhesión de los cementos resinosos para el cementado de postes ${ }^{[14]}$. Por lo tanto se podría especular que los irrigantes endodónticos sean los que interfieren en la adhesión y no el eugenol. Aunque si la controversia continúa sería interesante realizar estudios en esa dirección.

Ferreto y col. en 2006 estudiaron el efecto de varios cementos temporales en la fuerza de adhesión a dentina en adhesivos dentinales de una y dos botellas; del cual concluyeron que los cementos con eugenol no afectan significativamente la fuerza de adhesión cuando se comparan con cementos temporales sin eugeno[ $^{[15]}$. Aunque este estudio no se realizó en dentina intracameral se puede extraer que el efecto de un contaminante no interfiere en la adhesión de los sistemas adhesivos, al igual que los resultados obtenidos por Loaiza en dentina profunda radicular. Caso contrario es el presente estudio donde los irrigantes endodónticos si afectan la fuerza de adhesión.

Actualmente, cuando se restauran dientes endodónticamente tratados y sobre todo severamente destruidos se ve la necesidad de la utilización de postes intraradiculares con cementos resinosos, confección de muñones, coronas entre otros; para estos tratamientos se debe recurrir a la adhesión sobre una dentina que previamente se encontraba embebida con irrigantes endodónticos -mientras se realizaba los tratamientos de conductos-, en contacto con cementos de diferente naturaleza, en fin la adhesión se debe hacer sobre dentina contaminada con diferentes sustancias ${ }^{[14]}$. Estas circunstancias pueden llevar al fracaso a cualquier tratamiento restaurativo, ya sea una restauración con resina, una incrustación, un poste y/o una corona. Por lo tanto este estudio brinda un punto de vista que podría ayudar a superar los problemas adhesivos que se pueden presentar en estos casos.

\section{CONCLUSIONES.}

Dentro de las condiciones en las que se realizó el presente estudio se puede concluir:

1. La fuerza de adhesión microtensil de los adhesivos Single Bond 2, One Step Plus y PQ1 fueron estadísticamente iguales entre sí para el mismo irrigante.

2. Cuando se trata la dentina intracameral con irrigantes endodónticos como el $\mathrm{NaOCl} 3 \%$ o
$\mathrm{NaOCl} 3 \%$ + EDTA 17\% en combinación; la fuerza de adhesión microtensil de los adhesivos Single Bond 2, One Step Plus y PQ1 disminuye.

\section{AGRADECIMIENTOS.}

- MSc. David Lafuente. Prostodoncia, Catedrático Facultad de Odontología, Universidad de Costa Rica (UCR). San José-Costa Rica.

- Dra. Tatiana Ramírez. Especialista en Endodoncia. Profesor departamento endodoncia, Universidad de Costa Rica (UCR). San José-Costa Rica.

\section{REFERENCIAS BIBLIOGRÁFICAS}

1. Vongphan, N., Senawongse, P., Somsiri, W., Harnirattisai, C. , (January 2005) "Effects of sodium ascorbate on microtensile bond strength of totaletching adhesive system to $\mathrm{NaOCl}$ treated dentine". J. Dent, Vol. 33, 689-695.

2. Belli, S., Zhang,Y., Pereira, P., Ozer, F., Pashley, D.H. , (August 2001) "Regional bond strengths of adhesive resins to pulp chamber dentin". J. Endod, Vol. 27, N. 8.

3. Ozturk, B., Özer, F. (May 2004) "Effect of $\mathrm{NaOCl}$ on Bond Strengths of Bonding Agents to Pulp Chamber Lateral Walls". J. Endod, Vol. 30, N. 5.

4. Henostroza, G., (2003) "Adhesión en Odontología Restauradora". Libro. Primera Edición. Brasil: Editora Maio.

5. Scotti, R., (2004) Ferrari, M. "Pernos de fibra. Bases teóricas y aplicaciones clínicas". Libro. Editorial Masson. Cap.1.

6. Nascimento, J.S., Oliveira, M.R, de Goes, M.F., Zaia, A., Figueiredo, B., Souza-Filho, F., Randi, C., (November 2006) "Effect of chemical irrigants on the bond strength of a self-etching adhesive to pulp chamber dentin". J. Endod, Vol. 32, N. 11.

7. Belli, S., Zhang,Y., Pereira, P.N.R., Pashley, D.H., (August 2001) "Adhesive sealing of the pulp chamber". J. Endod, Vol. 27, N. 8.

8. Pashleyl, D.H., Sanoz, H., Ciucchis, B., Yoshiyamad, M., Carvalhos, R.M. (March 1995) "Adhesion testing of dentin bonding agents: A review". Dental Mat. Vol. 11:117-I 25. 
9. Dental materials - Testing of adhesión to tooth structure. ISO/TS 11405. (2003) Especificación técnica Tinius Olsen H10K-S.

10. Soares, I.J., Goldberg, F. (Abril 2003) "Endodoncia, técnica y fundamentos". Editorial médica panamericana.

11. Sayin, T.C., Serper, A., Cehreli, Z.C., Otlu, H.G. (2007) "The effect of EDTA, EGTA, EDTAC, and tetracycline- $\mathrm{HCl}$ with and without subsequent $\mathrm{NaOCl}$ treatment on the microhardness of root canal dentin". Oral Surg Oral Med Oral Pathol Oral Radiol Endod. ;104:418-24.

12. Sanches, B.A.F., Bittar, A.R., Pascon, F.M., Sobrinho, L.C., Martin, A.A., Puppin, R.M. (2008) "NaOCl effects on primary and permanent pulp chamber dentin" J.Dent. Vol. 36, 745-753.

13. Saleh, A.A., Ettman, W.M. (1999) "Effect of endodontic irrigation solutions on microhardness of root canal dentine". J.Dent. 27, 43-46.

14. Loaiza, A.E. (2006) "Efecto de los cementos eugenólicos para endodoncia en la adhesión del cemento de resina". Estudio Piloto. Universidad de Costa Rica. Trabajo final de graduación.

15. Ferreto, G. I., Lafuente, M.D., Gutiérrez, M.N. (2006) "Efecto de varios cementos temporales en la fuerza de adhesión a dentina en adhesivos dentinales de una y dos botellas". Estudio piloto. Universidad de Costa Rica. Odonvtos. Vol. 8. Pag. 91. 